\title{
Effect of an axial throughflow on buoyancy-induced flow in a rotating cavity
}

\author{
Diogo B. Pitz ${ }^{\mathrm{a}, *}$, John W. Chew, Olaf Marxen \\ Thermo-Fluid Systems University Technology Centre \\ University of Surrey \\ GU2 7XH Guildford, United Kingdom \\ ${ }^{a}$ Current address: \\ Faculty of Mechanical Engineering \\ University of Campinas \\ Rua Mendeleyev, 200 - Cidade Universitária \\ 13083-860 Campinas, Brazil
}

\begin{abstract}
In this paper large-eddy simulation is used to study buoyancy-induced flow in a rotating cavity with an axial throughflow of cooling air. This configuration is relevant in the context of secondary air systems of modern gas turbines, where cooling air is used to extract heat from compressor disks. Although global flow features of these flows are well understood, other aspects such as flow statistics, especially in terms of the disk and shroud boundary layers, have not been studied. Here, previous work for a sealed rotating cavity is extended to investigate the effect of an axial throughflow on flow statistics and heat transfer. Time- and circumferentially-averaged results reveal that the thickness of the boundary layers forming near the upstream and downstream disks is consistent with that of a laminar Ekman layer, although it is shown that the boundary layer thickness distribution along the radial direction presents greater variations than in the sealed cavity case. Instantaneous profiles of the radial and azimuthal velocities near the disks show good qualitative agreement with an Ekman-type analytical solution, especially in terms of the boundary layer thickness. The shroud heat transfer is shown to be governed by the local centrifugal acceleration and by
\end{abstract}

\footnotetext{
*Corresponding author

Email address: pitz@fem.unicamp.br (Diogo B. Pitz )
} 
a core temperature, which has a weak dependence on the value of the axial Reynolds number. Spectral analyses of time signals obtained at selected locations indicate that, even though the disk boundary layers behave as unsteady laminar Ekman layers, the flow inside the cavity is turbulent and highly intermittent. In comparison with a sealed cavity, cases with an axial throughflow are characterised by a broader range of frequencies, which arise from the interaction between the laminar jet and the buoyant flow inside the cavity.

Keywords: rotating cavities, buoyancy-induced flow, axial throughflow, large-eddy simulation

\section{Introduction}

Modern jet engines used in civil aviation operate at high pressure ratios and high turbine inlet temperatures. While these two requirements contribute to increasing the engine efficiency, they impose severe operating conditions on 5 the materials used. To maintain the temperature of key components within the material limits and to control thermal stresses, the internal, or secondary, air system is critical in modern engine designs. In these systems, air is bled from a certain compressor stage towards the core of the engine, where it flows through the disk cavities formed between the compressor disks, extracting heat from them in the process, and is further employed to cool other components, such as turbine blades and disks. It is important to note that the air used within the secondary air system is parasitic, in the sense that it does not directly contribute to generate power, therefore the air system must operate as efficiently as possible. For an overview of secondary air systems, see ref. [1].

Figure 1 shows a schematic representation of air flow through an internal air system, in the compressor region. During most operating conditions, the axial throughflow air extracts heat from the compressor disks. The degree of heat transfer affects thermal stresses in the disks and running clearances between the compressor blades and the outer casing. These are critical aspects of design affecting component life and compressor efficiency. 


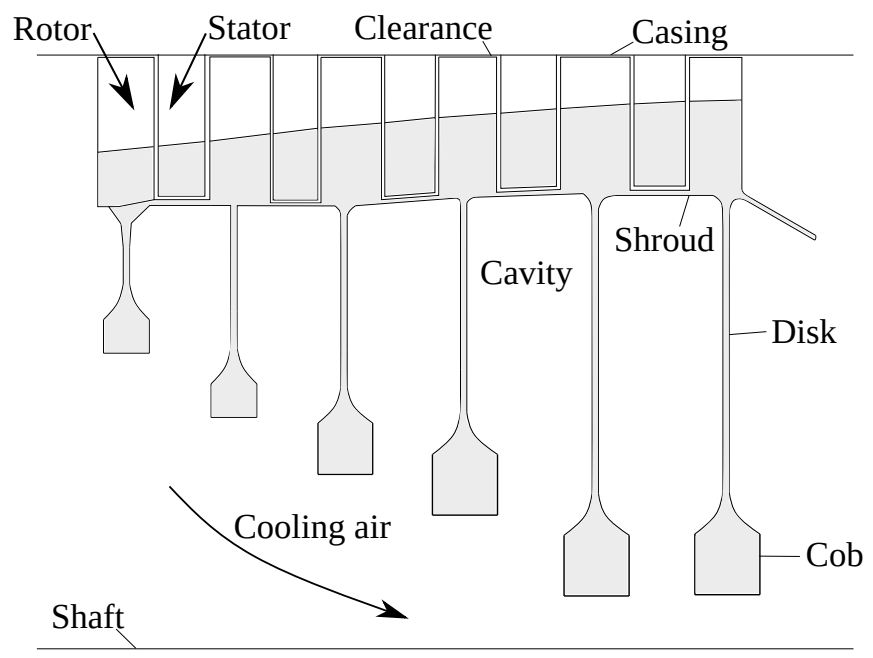

Figure 1: Schematic representation of the compressor region of a gas turbine secondary air system.

Since the compressor disks rotate at the same angular velocity, there is no mean azimuthal shear in the main part of the inter-disk cavities. Hence the flow is strongly affected by buoyancy in the centrifugal force field. Note that the axial throughflow can penetrate the cavity both due to buoyancy effects and to vortex breakdown, although experimental results for isothermal cavities (Owen \& Pincombe [2, Farthing et al. 3]) suggest that at the range of Rossby numbers relevant to engine conditions no vortex breakdown occurs, thus buoyancy effects dominate the dynamics. The Rossby number is defined as the ratio of the axial throughflow velocity to a characteristic tangential velocity. Since buoyancy-induced flows are inherently unsteady and, in the case of a rotating cavity, three-dimensional, they are challenging to predict numerically using the approaches commonly used in industry, which generally utilise RANS (Reynolds-averaged Navier-Stokes)-based turbulence models. For this reason, some numerical studies have employed large-eddy simulation (LES) techniques to investigate buoyant flows in rotating cavities.

The experiments of Farthing et al. 3, 4, revealed that fluid from the axial jet enters the cavity through thin radial arms, and that large-scale, counter- 
rotating cells span a great extent of the cavity, creating regions of low and high pressure. Later, Long et al. [5] observed a similar flow pattern in their experiments, and concluded that at low radii the flow was strongly affected by the axial jet, whereas close to the shroud it was dominated by buoyancy effects. Subsequently, Long \& Childs [6] concluded that the shroud Nusselt number could be estimated using correlations valid for natural convection under gravity, with the centrifugal acceleration playing a role analogous to that of gravity. These global

${ }_{45}$ aspects observed experimentally were reproduced with some success using numerical simulations (e.g. refs. [3, 7, 8, 9, 10, 11, 12]). Still, it is important to note that time-averaged statistics have not been reported in previous studies and that some aspects of the flow physics are not fully understood. Velocity and temperature profiles, as well as their respective fluctuations, can help to elucidate, for instance, the nature of the Ekman layers forming near the disks and of the shroud thermal boundary layer, and also how the axial jet interacts with the otherwise purely buoyant flow inside the cavity.

Another question discussed in recent publications is whether the flow in compressor disk cavities is laminar or turbulent. Although experimental and numerical observations suggest that the flow is highly unsteady and three-dimensional, a theoretical model proposed by Owen \& Tang [13, 14, where the Ekman layers are assumed to be laminar, was successfully matched to disk temperature and heat transfer rates obtained experimentally by Atkins \& Kanjirakkad [15]. In the present work energy spectra obtained from numerical probes and comparisons of near-disk velocity profiles with laminar Ekman layer solutions are used to obtain further insight into this issue.

The complexity of buoyancy-induced flows has motivated the use of simplified models to investigate the basic flow dynamics. In particular, the model of a sealed cavity, i.e. without throughflow, has been used in several numeri65 cal studies focused on understanding fundamental aspects of these flows (e.g. [16, 17, 18, 19, 20, 21]). In our recent work [20], we have used LES to obtain time- and circumferentially-averaged profiles of mean and fluctuating quantities for a sealed rotating cavity. By analysing profiles of mean and r.m.s radial veloc- 
ity, it has been shown that the disk boundary layers behave as highly unsteady laminar Ekman layers. The results revealed that, even instantaneously, these boundary layers can be approximated by an analytical solution for a laminar Ekman layer with a non-zero core radial velocity. Analyses of the shroud thermal boundary layer and Nusselt number for different Rayleigh numbers $(R a)$ indicated a scaling behaviour consistent with high- $R a$ natural convection under gravity. The results also show that the value of the centrifugal Rayleigh number has a weak effect on the core temperature inside the cavity.

Motivated by these findings, the objective of the present paper is to study the influence of an axial throughflow on the flow statistics obtained for a sealed cavity. To accomplish this, the geometry used in [20] is adapted to include an axial throughflow, so that the statistics obtained for the two geometries can be directly compared.

The paper is organised as follows. In section 2 the geometry, boundary conditions, non-dimensional parameters and governing equations are presented. A brief description of the numerical method used is also given, and a gridindependence study is performed to assess the quality of the numerical solutions. In section 3 the results for a rotating cavity with an axial throughflow are presented, including comparisons with the case of a sealed cavity. Conclusions and suggestions for future work are given in section 4 .

\section{Mathematical formulation}

90 2.1. Problem description

Consider air with kinematic viscosity $\nu=1.5 \times 10^{-5} \mathrm{~m}^{2} / \mathrm{s}, \operatorname{Pr} \equiv \nu / \alpha=0.7$, where $\alpha$ is the thermal diffusivity, at temperature $T=T_{i}$ flowing through a rotating annular pipe of inner and outer radii $r_{s}$ and $a$, respectively, at a mean axial velocity $W$, entering a rotating cavity at $z=0$ and exiting the cavity into

95 an outlet pipe at $z=d$. The lengths of the inlet and outlet pipes are equal to $1.1 d$, and the outer radius of the cavity is $b$. This configuration is illustrated schematically in Fig. 2(a). For comparison, Fig. 2(b) shows the geometry of 
a sealed rotating cavity used in our previous study [20], which is based on the experimental configuration used by Bohn et al. [22]. The dimensions are such that $a=0.125 \mathrm{~m}, b=0.24 \mathrm{~m}$ and $d=0.12 \mathrm{~m}$, and the shaft of the cavity with axial throughflow has a radius $r_{s}=0.115 \mathrm{~m}$.

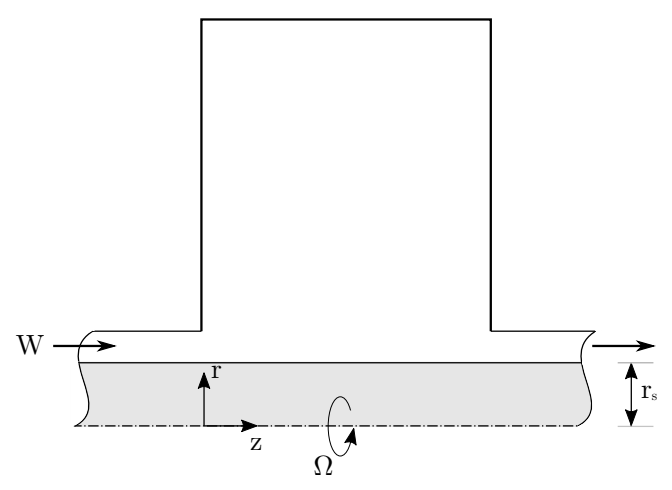

(a)

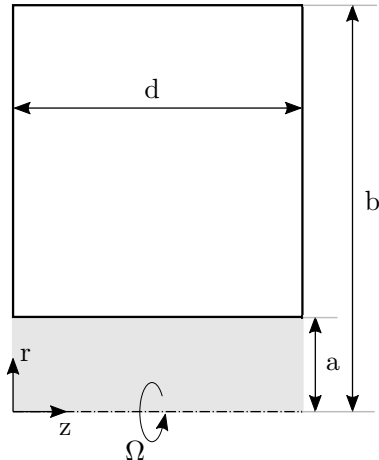

(b)

Figure 2: Schematic representation of (a) a rotating cavity with an axial throughflow of cooling air and (b) a sealed cavity.

The flow at the inlet pipe is assumed to be laminar, since this significantly reduces the computational cost of the simulations. Thus, at the inlet a laminar velocity profile for flow in a non-rotating annular pipe is imposed, whereas at the outlet the robust outflow boundary condition proposed by Dong et al. [23] is applied. Within this formulation, a Neumann boundary condition is used for velocities and a Dirichlet condition is used for pressure, however the prescribed values are calculated such that any energy entering the domain (reverse flow) is balanced by an imposed stress. This allows the use of a rather short outlet pipe, without requiring the flow to be fully developed while still preserving numerical stability. On the solid surfaces, no-slip boundary conditions are applied to all velocity components.

The walls of both the inlet and outlet pipes are adiabatic, and the shroud is kept at a constant temperature $T_{b}$, which is higher than the axial throughflow temperature $T_{i}$, such that the stratification is unstable due to the centrifugal 
force field. The side-wall disks are either adiabatic or heated. For the latter case, the temperature profile shown in Fig. 3 is imposed. This was obtained from an interpolation of a set of experimental data reported by Atkins \& Kanjirakkad [15, so that it is representative of an experimental condition. Note that, in Fig. ${ }_{120} 3 r^{*}=(r-a) /(b-a)$ and $T^{*}=\left(T-T_{i}\right) /\left(T_{b}-T_{i}\right)$ denote the non-dimensional radial coordinate and temperature, respectively.

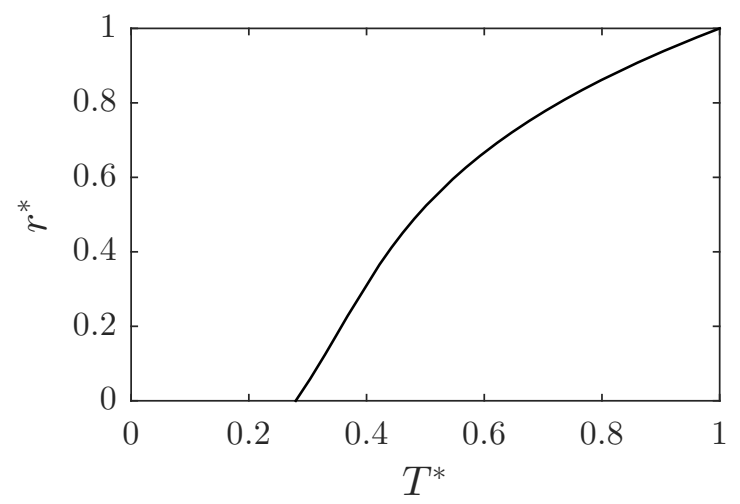

Figure 3: Temperature profile applied to the upstream and downstream disks for the cases where the disks are heated.

The flow is characterised by the centrifugal Rayleigh number $R a$, the rotational Reynolds number $R e$, the axial Reynolds number $R e_{z}$ and the Rossby number Ro, given, respectively, by,

$R a=\frac{\Omega^{2} r_{m} \beta \Delta T(b-a)^{3}}{\nu \alpha}, \quad R e=\frac{\Omega r_{m}(b-a)}{\nu}, \quad R e_{z}=\frac{2\left(a-r_{s}\right) W}{\nu}, \quad R o=\frac{W}{\Omega a}$,

where $r_{m}=(b+a) / 2$ is the cavity mean radius, $\beta$ is the thermal expansion coefficient and $\Delta T=T_{b}-T_{i}$ is the temperature difference between the shroud and the throughflow jet. For a fixed geometry, $R a, R e$ and $R e_{z}$ are sufficient to fully characterise the flow, since $R o$ can be written as a combination of $R e$ and $R e_{z}$. We consider a single Rayleigh number, $R a=10^{8}$, and two axial 130 Reynolds numbers, $R e_{z}=1000$ and $R e_{z}=2000$. It should be noted that these values of $R e_{z}$ are significantly lower than those encountered in engine conditions, which are in the turbulent regime. Although a turbulent inlet would modify 
the nature of the interaction between the axial throughflow and the buoyancydriven flow inside the cavity, the assumption of a laminar throughflow serves as a first step in investigating such interactions, with a reduced computational cost. Note that when the angular speed is varied to change the Rayleigh number, the rotational Reynolds number is also affected. Here, the correlation between $R a$ and $R e$ considered in the experiments of Bohn et al. [22] is employed, $R e=1.441 R a^{0.557}$. While the Rayleigh and Reynolds numbers used are below engine conditions, the resulting Rossby numbers, which relate the mean axial throughflow velocity with the rotational speed at the cavity inner radius, are within the range $0.1-1$, which is consistent with values encountered during engine operation.

\subsection{Governing equations and numerical method}

The flow is governed by the Navier-Stokes equations written in incompressible form, with density variations accounted for using the Boussinesq approximation. The assumption of incompressibility is valid for low-Mach number flows, and within the Boussinesq approximation it is assumed that the density $\rho$ is constant, except if it is multiplying a term that induces buoyancy, in which case $\rho=\rho(T)=\rho_{0}+\rho^{\prime}=\rho_{0}\left[1-\beta\left(T-T_{0}\right)\right]$, where $\rho_{0}$ and $\rho^{\prime}$ denote a reference density and its fluctuation, respectively. With these considerations, the governing equations are,

$$
\begin{gathered}
\nabla \bullet \mathbf{u}=0, \\
\frac{\partial \mathbf{u}}{\partial t}+\mathbf{u} \bullet \nabla \mathbf{u}=-\frac{1}{\rho_{0}} \nabla p^{*}+\nu \nabla^{2} \mathbf{u}-2 \boldsymbol{\Omega} \times \mathbf{u}+\beta\left(T-T_{0}\right) \boldsymbol{\Omega} \times(\boldsymbol{\Omega} \times \mathbf{r}), \\
\frac{\partial T}{\partial t}+\mathbf{u} \bullet \nabla T=\alpha \nabla^{2} T,
\end{gathered}
$$

where $\mathbf{u}=\left(u_{r}, u_{\theta}, u_{z}\right)$ is the velocity vector, $p^{*}$ denotes a reduced pressure, $\mathbf{r}$ is the position vector and $T$ is the temperature field. Note that, since the 
equations are solved in a non-inertial reference frame rotating at a constant angular velocity $\boldsymbol{\Omega}$, the Coriolis and centrifugal forces enter the equations explicitly. As the entire system rotates at the same angular frequency, the three velocity components are set to zero on all solid surfaces. The leading centrifugal force component can be written as the gradient of a scalar, and is absorbed into the pressure gradient term, but a variable density component of the centrifugal force must be retained, giving the last term in Eq. 2 .

The governing equations are solved numerically using a nodal spectral elementFourier method formulated in cylindrical coordinates, described by Blackburn \& Sherwin 24 . Since the geometry considered is homogeneous along the azimuthal direction, it is discretised using a Fourier series decomposition, whereas the radial-axial plane is discretised with quadrilateral spectral elements. In spectral element methods, the Galerkin method commonly employed in finite element formulations is used, but high-order polynomial expansion bases are utilised. This allows the grid to be refined by either reducing the element size ( $h$ refinement) or by increasing the polynomial order ( $p$ refinement). Such methods have favourable properties in the context of numerical simulation of turbulent flows, particularly for long time-integration periods (Karniadakis \& Sherwin [25]). For time discretisation, a semi-implicit, second-order, stiffly stable scheme is used (Karniadakis et al. [26]). For further details of the numerical method, 175 the reader is referred to refs. [24, 25, 26]. For previous applications of the method involving rotating flows with centrifugal buoyancy, see refs. [18, 19, 20].

The large-eddy simulation (LES) technique used consists of adding a controlled amount of artificial dissipation to the viscous term at high wavenumbers, so that no explicit sub-grid scale model is employed. In the context of spectral and spectral element formulations, this technique is known as spectral vanishing viscosity (SVV), and can be interpreted as a numerical stabilisation procedure. If the grid is not fine enough to resolve all the relevant flow scales, energy will pile up at high wavenumbers, therefore it is reasonable to augment the viscosity to avoid uncontrolled energy growth. To implement the technique, it is necessary to modify the viscous term only during pre-processing, which implies that 
the computational cost of a simulation is not affected when SVV is activated. The stabilisation is applied in all directions. Along the azimuthal coordinate, it is applied in Fourier (spectral) space, whereas in the radial and axial directions the viscosity is augmented in polynomial space, applying a discrete polynomial transform. An exponential kernel, such as that employed by Karamanos \& Karniadakis [27, is used to apply an increasing amount of dissipation as the wavenumber increases. In particular, it is necessary to impose the amplitude of the dissipation $\epsilon$ as well as a wavenumber cut-off in both the Fourier and spectral element discretisations, denoted as $M_{\theta}$ and $M_{P}$, respectively. For details of the technique and applications, see refs. 27, 28, 29].

\subsection{Details of the computations}

For all computations, the mesh used is formed of 452 spectral elements in the $r-z$ plane, as shown in Fig. 4 Note that, within each element, high-order polynomials are used to approximate the solution, and for this reason element sizes in spectral element grids are much larger than those used in low-order discretisation schemes. The grid design is based on the strategy used in our previous work for a sealed rotating cavity (Pitz et al. [20]), where the nearwall spacing was such that there were 10 degrees of freedom within the shroud thermal boundary layer and 10 degrees of freedom within the disk boundary layers, when a polynomial of order $P=7$ was used, for $R a=10^{8}$. Note that, since the flow at the inlet pipe is laminar, the resolution in this region can be significantly relaxed. If the flow in this region was turbulent, then a significant amount of computational effort would be spent to properly resolve it, since the mesh would have to be more refined and, additionally, it was verified that the time step used would have to be significantly smaller, thus making the computations very expensive.

To assess grid-independence, the case $R e_{z}=1000, R a=10^{8}$ with adiabatic disks was considered. For a coarse mesh, the polynomial order is fixed at $P=7$ and the number of planes in the azimuthal direction is $N_{\theta}=96$, whereas for a 


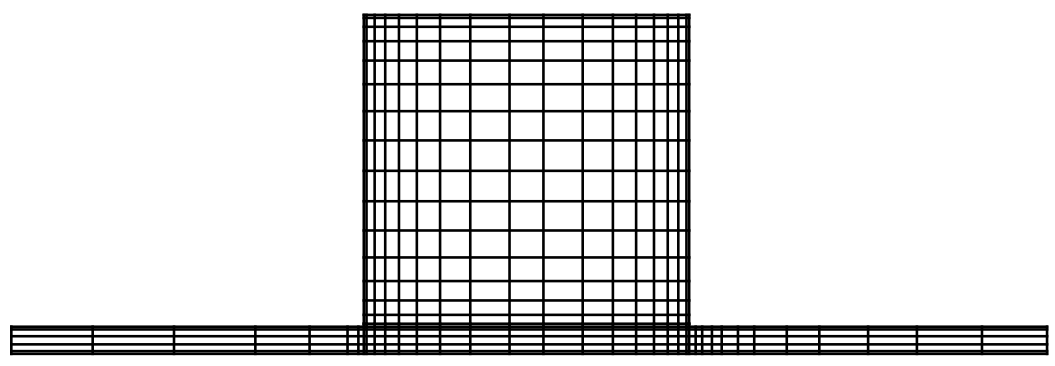

Figure 4: Mesh with 452 spectral elements used in the computations.

freedom than the coarse mesh. In all cases, the SVV amplitude is set to $\epsilon=4 \nu$. For the coarse mesh, $M_{\theta}=24$ and $M_{P}=5$, and for the fine mesh $M_{\theta}=64$ and $M_{P}=6$. A sensitivity study of the SVV parameters on the solution is out of the scope of this paper, however these values were chosen based on recommendations from previous studies (e.g. Koal et al. [29]).

Table 1 shows the effect of $P$ and $N_{\theta}$ on key quantities analysed in the paper. The results were obtained from time- and circumferentially-averaging the solution for at least 10 disk revolutions, after a statistically steady state based on the core temperature and shroud Nusselt number was reached. To extract the core temperature, the solution was averaged along the axial direction, $0 \leqslant$ $z^{*} \leqslant 1.0$, and only a limited radial section, $0.4 \leqslant r^{*} \leqslant 0.6$, was averaged to obtain the value of $T_{\text {core }}^{*}$. Note that the non-dimensional axial coordinate is defined as $z^{*}=z / d$. The disk kinetic boundary layer thickness $\delta_{r m s}^{*}=\delta_{r m s} / d$ was calculated based on the distance from the upstream disk where the radial velocity fluctuation $u_{r, r m s}$ peaks, and averaged over the interval $0.2 \leqslant r^{*} \leqslant 0.8$ so as to exclude the region strongly influenced by the shroud and the axial jet. $\lambda_{r m s}^{*}=\lambda_{r m s} /(b-a)$ represents the shroud thermal boundary layer thickness based on the peak of $T_{r m s}$, and was calculated from an average along $0.2 \leqslant$ $z^{*} \leqslant 0.8$. Finally, the shroud Nusselt number shown in Table 1 was evaluated by computing the heat flux $q_{b}$ on the shroud surface and dividing it by the heat 
Table 1: Effect of the polynomial order $P$ and number of azimuthal planes $N_{\theta}$ on $T_{\text {core }}^{*}, \delta_{r m s}^{*}$, $\lambda_{r m s}^{*}$ and $N u$, for $R a=10^{8}$ and $R e_{z}=1000$ on the mesh with $N_{e l}=452$ spectral elements shown in Fig. 4

\begin{tabular}{cccccc}
\hline$P$ & $N_{\theta}$ & $T_{\text {core }}^{*}$ & $\delta_{r m s}^{*}$ & $\lambda_{r m s}^{*}$ & $N u$ \\
\hline 7 & 96 & 0.2312 & 0.01768 & 0.01394 & 64.51 \\
9 & 256 & 0.2260 & 0.01768 & 0.01516 & 62.09 \\
\hline
\end{tabular}

flux due to conduction in the sealed cavity case, i.e.,

$$
N u=\frac{q_{b} b \log (b / a)}{k \Delta T} .
$$

Although only two meshes are considered, it is clear that the quantities analysed are only weakly affected by the change in resolution. As observed in the case of a sealed cavity [20, the shroud boundary layer thickness is the most sensitive of the quantities, while the disk kinetic boundary layer thickness is effectively independent of the resolution. To have better reliability on the values of $\lambda_{r m s}^{*}$, the mesh with $P=9$ and $N_{\theta}=256$ was employed in the remaining calculations. In previous work for a sealed cavity [20, further mesh refinement along the azimuthal direction had no significant effect on the value 245 of $\lambda_{r m s}^{*}$.

\section{Results and discussion}

To give a first impression of how the presence of an axial throughflow affects the flow structure inside the cavity, Fig. 5 shows contours of the normalised temperature $T^{*}$ for the cases of a sealed cavity, $R e_{z}=1000$ and $R e_{z}=2000$. In 250 all cases the snapshots were taken at the mid-axial position $z^{*}=0.5$, and the disks are adiabatic. For the sealed cavity the temperature is nearly constant except near the boundary layers and in radial arms, or plumes, emanating from these layers. There is a rather weak structure comprising pairs of counterrotating cells with radial arms of cold or hot fluid moving outwards or inwards, 

behaviour, although for the latter case the cold fluid penetrates further radially into the cavity.

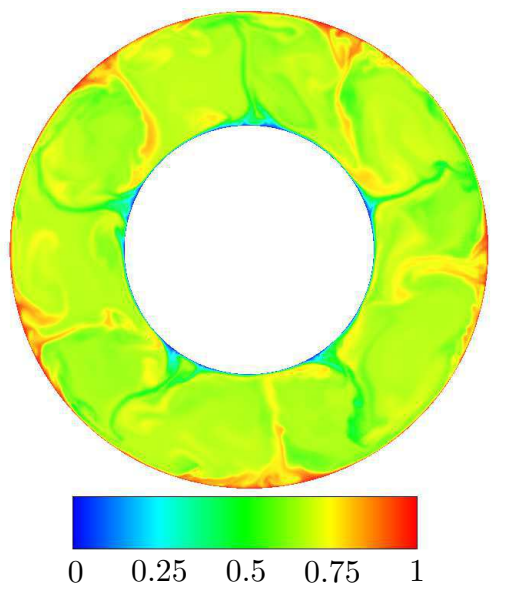

(a) sealed cavity

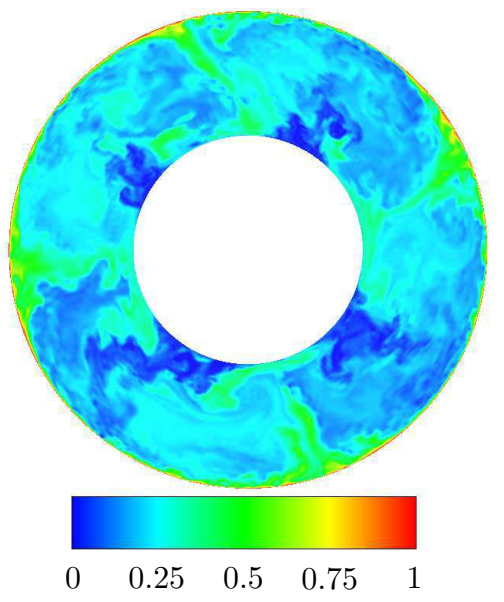

(b) $R e_{z}=1000$

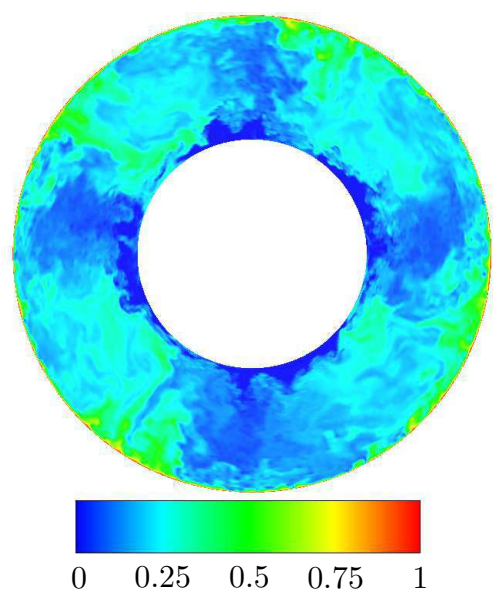

(c) $R e_{z}=2000$

Figure 5: Instantaneous contours of normalised temperature $T^{*}$ at the mid-axial position for $R a=10^{8}$ with adiabatic disks. The cavities rotate in the counter-clockwise direction.

An analysis of time- and circumferentially-averaged contours in the $r-z$ plane shows that the temperature along the axial direction has a rather different behaviour between the two cases - see Fig. 6(a). First, note that for the sealed cavity case the temperature is quasi-uniform along the axial direction, and the contours are symmetric relative to $z^{*}=0.5$. Also, the axial velocity, here denoted by $u_{z}^{\star}=u_{z} /(\Omega a)$, is very small, as shown in Fig. 6(b). When the axial throughflow is included the flow loses its near-homogeneity and symmetry along the axial direction. An increase of $T^{*}$ with $z^{*}$ occurs for $R e_{z}=1000$, whereas 
for $R e_{z}=2000$ the opposite occurs. The contours of axial velocity shown in Fig. 6(b) help with the interpretation of these results. For $R e_{z}=1000$ the axial jet is almost immediately broken, in the sense that its axial velocity reduces significantly, when it enters the cavity. The cold fluid entering the cavity is then pulled radially outwards by buoyancy effects, which causes a greater reduction in the temperature in the left part of the cavity. For $R e_{z}=2000$ the cold fluid entering the cavity has more momentum and penetrates further axially into the cavity before it is significantly affected by the buoyant force. Thus, the temperature reduction inside the cavity is greater on the right-hand side.

\subsection{Kinetic boundary layers}

An analysis of averaged fields in terms of fluctuating quantities shows similarities and differences with the case of a sealed cavity reported in ref. 20]. Near each of the disks, a kinetic boundary layer can be defined in terms of the peak of the radial velocity fluctuation $u_{r, r m s}^{*}$. Figure $7($ a) shows near-disk profiles of $u_{r, r m s}^{*}$ at $r^{*}=0.5$ for the sealed cavity and for the two axial throughflow cases with adiabatic disks. Although all the curves are similar, it is clear that the presence of an axial throughflow increases the magnitude of the velocity fluctuations, which is not surprising considering the fact that, in these cases, cold fluid is fed continually into the cavity, whereas in the sealed cavity case the fluid circulation inside the cavity occurs due to natural convection only. Despite the qualitative differences in the flow structure between the cases $R e_{z}=1000$ and $R e_{z}=2000$, the profiles are nearly identical at the radial station considered. Similarly, as in Fig. 7(b), a radial profile of $u_{r, r m s}^{\star}$ averaged along the entire axial extent of the cavity $\left(0 \leqslant z^{*} \leqslant 1\right)$ also reveals that the presence of the axial throughflow increases the fluctuations in comparison with the sealed cavity case. The profiles are again rather similar for the two values of $R e_{z}$. Note that the axial profile $u_{r, r m s}^{*}$ is normalised with $\Omega r$, where $r$ is the local radius, whereas the radial profile $u_{r, r m s}^{\star}$ is normalised with $\Omega a$ so that its shape is not distorted. Also, note that the non-dimensional radial coordinate is defined as ${ }_{300} r^{*}=(r-a) /(b-a)$, and that is the reason why the region between the shaft 
and the inner disk radius has a negative $r^{*}$ in Fig. 7(b).

In contrast with the sealed cavity case, the cases with axial throughflow are not symmetric with respect to the $z^{*}=0.5$ plane, which means that the upstream and downstream disk layers may behave differently. Figures 8 (a) and (b) show the radial variation of the disk kinetic boundary layer thickness based on the peak of $u_{r, r m s}^{*}$ for the upstream and downstream disks, respectively, for the two values of $R e_{z}$ considered. The dashed and dot-dashed lines indicate the thickness of a laminar Ekman layer, $\pi \sqrt{\nu / \Omega}$, and the Ekman depth, $\sqrt{\nu / \Omega}$, respectively, while the open circles denote results obtained for a sealed cavity. The values of $\delta_{r m s}^{*}$ are overall slightly higher in the cases with an axial throughflow, but they are still consistent with the thickness of an Ekman layer. For $R e_{z}=1000 \delta_{r m s}^{*}$ is nearly constant along $r^{*}$ for both disks, but for $R e_{z}=2000$ much more significant variations occur. One possible explanation for the change in behaviour near the downstream disk for $R e_{z}=2000$ is that the axial jet is impinging on the disk. This is supported by the contours of temperature and axial velocity shown in Fig. 6, where it is clear that the cavity is colder near the downstream disk. In order to fully characterise the nature of the disk boundary layers, it would be necessary to consider higher values of $R e_{z}$, to assess whether this would promote more mixing inside the cavity, and also different values of the rotational Reynolds number $R e$ and Rayleigh number $R a$ (recall that here only $R a=10^{8}$ is studied), to analyse the scaling of $\delta_{r m s}^{*}$ with $R e$. The results presented here suggest, however, that the values of $\delta_{r m s}^{*}$ still scale consistently with a laminar Ekman layer. In previous work [20] for a sealed rotating cavity, it was observed that $\delta_{r m s}^{*}$ scales as a laminar Ekman layer within $10^{7} \leqslant R a \leqslant 10^{9}$.

In ref. 20] it was shown that, for a sealed cavity, the disk boundary layers behave as laminar Ekman layers even in the instantaneous sense. Defining a non-zero core radial velocity $u_{r, c o r e}$, as well as a core tangential velocity, $u_{\theta, c o r e}$, the analytical solution given by Eqs. 5 and 6 was compared with instantaneous 
profiles obtained numerically,

$$
\begin{aligned}
& u_{r, E k}=u_{r, \text { core }}\left(1-e^{-z / \delta} \cos \frac{z}{\delta}\right)-u_{\theta, c o r e} e^{-z / \delta} \sin \frac{z}{\delta}, \\
& u_{\theta, E k}=u_{\theta, \text { core }}\left(1-e^{-z / \delta} \cos \frac{z}{\delta}\right)+u_{r, \text { core }} e^{-z / \delta} \sin \frac{z}{\delta},
\end{aligned}
$$

330

where $\delta=\sqrt{\nu / \Omega}$ is the Ekman depth. This solution assumes the flow to be a small perturbation from uniform rotation. Figures 9 and 10 show analogous profiles obtained for the axial throughflow cases near the upstream and downstream disks, respectively. Note that the values of $\theta$ were chosen to illustrate regions where the numerical solution is and is not consistent with the laminar Ekman solution. One difficulty imposed by the axial throughflow is that the flow is not homogeneous along $z$, which makes it difficult to define a core velocity. Here the core velocities were obtained by averaging over $0.1 \leqslant z^{*} \leqslant 0.2$ (upstream disk) or $0.1 \leqslant 1-z^{*} \leqslant 0.2$ (downstream disk). Still, the profiles are overall consistent with the Ekman solution in terms of the thickness of the boundary layer. It

appears that adjustment of the core velocity could give better agreement with the analytical solution in the boundary layer region. Note that the range of $z^{*}$ shown in the plots is very narrow, up to $z^{*}=0.05$ and $1-z^{*}=0.05$ for the upstream and downstream disks.

\subsection{Temperature profiles and heat transfer}

Although the instantaneous contours of Fig. 5 revealed that when an axial throughflow is present the temperature across the cavity is less uniform than in a sealed system, when the results are time- and circumferentially-averaged a core of constant temperature is still observed, as shown in Fig. 11(a). As expected, the cooling air entering the cavity causes a dramatic reduction in the core temperature, however it is interesting to note that the value of $R e_{z}$ has little to no effect on the results in the core.

The existence of a core temperature motivates a comparison of the shroud heat transfer with correlations for natural convection over a horizontal heated 
flat plate. A modified Nusselt number $N u^{\prime}$, which depends on the core temperature, is defined by Eq. 7, and a modified Rayleigh number $R a^{\prime}$, defined in terms of the local centrifugal acceleration at $r=b$, is given by Eq. 8 ,

$$
\begin{gathered}
N u^{\prime}=\frac{q_{\text {conv }}(d / 2)}{k\left(T_{b}-T_{\text {core }}\right)}, \\
R a^{\prime}=\frac{\Omega^{2} b \beta\left(T_{b}-T_{\text {core }}\right)(d / 2)^{3}}{\nu \alpha} .
\end{gathered}
$$

Using the definitions above, $N u^{\prime}$ can be compared with correlations for natural convection over a heated horizontal flat plate obtained by Lloyd \& Moran [30. for low and high $R a^{\prime}$, which are given by

$$
\begin{gathered}
N u^{\prime}=0.54 R a^{1 / 4}, \quad 10^{4} \lesssim R a^{\prime} \lesssim 10^{7}, \\
N u^{\prime}=0.15 R a^{\prime 1 / 3}, \quad 10^{7} \lesssim R a^{\prime} \lesssim 10^{11} .
\end{gathered}
$$

Table 2 shows that $N u^{\prime}$ is only weakly affected by the value of $R e_{z}$, which is expected considering that the core temperature is very similar in the two cases. The table also shows the heat flux divided by the thermal conductivity, $q_{\text {conv }} / k$, indicating that the heat transfer increases significantly when an axial throughflow is included. Note that the two correlations for natural convection overestimate the numerical values. However, it should be noted that these correlations require only the local centrifugal acceleration and an estimate of the core temperature as input parameters. Also, the values of $R a^{\prime}$ used in the correlation are close to $10^{7}$, which corresponds to the transition region between the low- and high- $R a^{\prime}$ correlations.

Figure 11(b) shows that when an axial throughflow is included the temperature fluctuations are stronger near the shroud than in the inner part of the cavity, in contrast with the sealed cavity case. This occurs because the core temperature is significantly lower than $T^{*}=0.5$, which promotes more intense fluctuations in the shroud region. Conversely, this also promotes a reduction in the fluctuations at low $r^{*}$. 
Table 2: Modified shroud Nusselt number $N u^{\prime}$ for the case of adiabatic disks.

\begin{tabular}{ccccc}
\hline Case & $q_{\text {conv }} / k[K / m]$ & $N u^{\prime}$ & $N u^{\prime}\left(\right.$ low $\left.R a^{\prime}\right)$ & $N u^{\prime}\left(\right.$ high $\left.R a^{\prime}\right)$ \\
\hline Sealed & 160.2 & 26.84 & 27.46 & 28.26 \\
$R e_{z}=1000$ & 396.6 & 30.74 & 33.30 & 36.54 \\
$R e_{z}=2000$ & 407.2 & 31.62 & 33.28 & 36.52 \\
\hline
\end{tabular}

\subsection{Heated disks}

When the temperature profile shown in Fig. 3 is imposed on both the upstream and downstream disks, no major difference in the flow behaviour is observed. Figure 12 shows temperature and axial velocity contours for the sealed given by,

$$
N u_{d}=\frac{q r}{k\left(T_{d i s k}-T_{i}\right)},
$$

where $T_{\text {disk }}$ is the local disk temperature. For a sealed cavity, the profiles of $N u_{d}$ of the upstream and downstream disks are identical, but in the cases with axial throughflow the heat transfer is expected to be different between the disks, especially if jet impingement occurs. Figure 14 shows profiles of $N u_{d}$ on both disks for $R e_{z}=1000$ and 2000. For the lower value of $R e_{z}$, the two 
profiles are similar, indicating that jet impingement effects are rather weak. For $R e_{z}=2000$, in contrast, $N u_{d}$ reaches much higher values near the downstream disk and is positive for all $r^{*}$, i.e., the disk is losing heat to the cooling flow. This, together with the fact that the thickness of the kinetic boundary layer changes significantly with $r^{*}$ for $R e_{z}=2000$ (see Figs. 8 and 13), indicates that jet impingement effects are strong for this value of $R e_{z}$, even though this is not evident from the contours of axial velocity shown in Fig. 12. (b).

Figure 14 also shows plots of the modified disk Nusselt number $N u_{d}^{\prime}$, which 405 is given by,

$$
N u_{d}^{\prime}=\frac{\left(T_{\text {disk }}-T_{r, \text { core }}\right) r}{\delta_{0}\left(T_{\text {disk }}-T_{i}\right)},
$$

where $T_{r, \text { core }}$ is a radially local core temperature, defined as the averaged temperature along the axial direction, and $\delta_{0}$ is the thickness of a laminar Ekman layer, $\delta_{0}=\pi \sqrt{\nu / \Omega}$. Note that $N u_{d}^{\prime}$ is the same for both disks, as it is based on a core temperature. This simple expression gives the Nusselt number based on radius assuming heat transfer due to conduction between disk and core temperatures across the Ekman boundary layer. It is not able to reproduce the values of the disk Nusselt number with good accuracy, but provides a reasonable order-of-magnitude estimate of the disk heat transfer.

\subsection{Spectral analysis}

To analyse the differences between the flow features observed across the cavity, time series collected at specific locations are considered for the case of heated disks. First, the frequency contents of points located inside and outside the disk boundary layers are compared. Figure 15 shows, on the top row, time series of the normalised temperature $T^{*}$ as a function of the number of revolutions $t^{*}$ at $r^{*}=0.5, \theta=0$, for three axial positions: $z^{*}=2 \times 10^{-4}$, $z^{*}=0.02$ and $z^{*}=0.5$. It should be noted that for the point very close to the disk the temperature oscillates with a rather small amplitude in all cases, but the frequency content shown by the power spectral density (PSD) curves is similar to those obtained further from the disk. The point located at $z^{*}=0.02$ 
425 is immediately outside the boundary layer, as shown in Figs. 8 and 13 , and shows more significant oscillation amplitudes, comparable to those observed at $z^{*}=0.5$. For the sealed cavity, the oscillations observed are remarkably slow compared to the axial throughflow cases, which is reflected by the lack of highfrequency content shown in the PSD curves. Despite the relatively low frequency of the oscillations, they do present a chaotic behaviour, although the PSD does not seem to have a $f^{-5 / 3}$ region, which is characteristic of the inertial subrange in turbulent flows.

When an axial throughflow is present, the flow becomes much more chaotic and dominated by high-frequency oscillations, even very close to the upstream disk (Fig. 15(a)), and despite the fact that the axial throughflows considered are laminar. For both $R e_{z}=1000$ and $R e_{z}=2000$ the PSD plots reveal a $f^{-5 / 3}$ region, thus suggesting that the flow is fully turbulent - note that this occurs even at $z^{*}=2 \times 10^{-4}$.

Figure 16 shows temperature time histories at $r^{*}=0.98$, i.e. inside the shroud thermal boundary layer, and at $r^{*}=0.9$, or just outside the boundary layer, as well as the corresponding PSD curves. In this region, again, the presence of an axial throughflow increases the range of frequencies observed, as well as the amplitude of the temperature oscillations. The chaotic nature of the time series suggests that the heat transfer from the shroud to the cavity occurs via turbulent convection, even for the sealed cavity case.

To investigate the interaction between the axial jet and the cavity, Fig. 17 shows temperature time series at $r^{*}=0.04$ and at the mid-axial position. At this location the spectra of frequencies become even broader than at the other points analysed. It is interesting to note that the increase observed in the frequency range comes solely from the interaction between the jet and the cavity flow, and not from the jet itself, which is laminar at the cavity inlet. Previous studies suggested that buoyancy-induced flows in cavities with an axial throughflow can be split into a region of forced convection at low radii and a region dominated by natural convection, near the shroud. The results presented here show qualitatively that the jet has a strong interaction with the cavity flow 
at low radii, but also that it has a significant influence on the frequency content even very close to the shroud. As shown earlier, however, correlations commonly employed for natural convection are able to provide reasonable predictions for the shroud heat transfer. Therefore, while the present results indicate that the shroud region is dominated by natural convection, they also show that the axial throughflow has a strong effect on the temperature oscillations near the shroud.

\section{Conclusions}

In this paper LES was used to investigate the effect of an axial throughflow of cooling air on buoyant flow inside a rotating cavity. The geometry considered corresponds to an adaptation of the sealed cavity used in our previous work [20] to include an axial throughflow. By doing this, the effect of the axial jet on the flow structure and time-averaged statistics could be isolated and quantified.

Instantaneous contours revealed that the cold jet promotes a significant reduction in the temperatures inside the cavity in comparison with the sealed system. Large-scale structures comprised of radial arms also become more pronounced when the axial throughflow is included. Time- and circumferentiallyaveraged profiles of radial velocity fluctuation $u_{r, r m s}^{*}$ are consistent with those obtained for a sealed cavity, however the magnitude of this quantity is higher for the cases with axial throughflow due to the interaction of the axial jet with the flow inside the cavity. Still, the disk boundary layer thickness is consistent with that of a laminar Ekman layer for the value of $R a$ considered, near both the upstream and downstream disks. Instantaneous profiles of radial and tangential velocity extracted at different azimuthal positions are in reasonable agreement with a laminar Ekman solution which considers a non-zero radial velocity in the cavity core. Although the agreement of the numerical predictions with the analytical model is mostly qualitative (due to uncertainty in defining the core velocity), it is still remarkable, especially considering that the thickness of these instantaneous boundary layers agrees with the model.

Comparisons of the shroud heat transfer with correlations for natural con- 


\section{Acknowledgments}

D. B. P. acknowledges financial support from the CAPES foundation, grant no. $13507 / 13-3$

\section{References}

[1] J. W. Chew, N. J. Hills, Computational fluid dynamics for turbomachinery internal air systems, Philosophical Transactions of the Royal Society of 
London A: Mathematical, Physical and Engineering Sciences 365 (1859) (2007) 2587-2611.

[2] J. Owen, J. Pincombe, Vortex breakdown in a rotating cylindrical cavity, Journal of Fluid Mechanics 90 (01) (1979) 109-127.

[3] P. Farthing, C. Long, J. Owen, J. Pincombe, Rotating cavity with axial throughflow of cooling air: flow structure, Journal of Turbomachinery 114 (1) (1992) 237-246.

[4] P. Farthing, C. Long, J. Owen, J. Pincombe, Rotating cavity with axial throughflow of cooling air: heat transfer, Journal of Turbomachinery 114 (1) (1992) 229-236.

[5] C. Long, N. Miche, P. Childs, Flow measurements inside a heated multiple rotating cavity with axial throughflow, International Journal of Heat and Fluid Flow 28 (6) (2007) 1391-1404.

[6] C. Long, P. Childs, Shroud heat transfer measurements inside a heated multiple rotating cavity with axial throughflow, International Journal of Heat and Fluid Flow 28 (6) (2007) 1405-1417.

[7] M. Puttock-Brown, M. Rose, C. Long, Experimental and computational investigation of rayleigh-bénard flow in the rotating cavities of a core compressor, in: ASME Turbo Expo 2017: Turbomachinery Technical Conference and Exposition, American Society of Mechanical Engineers, 2017, pp. V05BT15A032-V05BT15A032.

[8] S. Tian, Z. Tao, S. Ding, G. Xu, Investigation of flow and heat transfer instabilities in a rotating cavity with axial throughflow of cooling air, in: ASME Turbo Expo 2004: Power for Land, Sea, and Air, American Society of Mechanical Engineers, 2004, pp. 373-380.

[9] S. Tian, Z. Tao, S. Ding, G. Xu, Computation of buoyancy-induced flow in a heated rotating cavity with an axial throughflow of cooling air, International Journal of Heat and Mass Transfer 51 (3) (2008) 960-968. 
[10] Z. Sun, K. Lindblad, J. W. Chew, C. Young, LES and RANS investigations into buoyancy-affected convection in a rotating cavity with a central axial throughflow, Journal of Engineering for Gas Turbines and Power 129 (2) (2007) 318-325.

[11] Q. Tan, J. Ren, H. Jiang, Prediction of flow features in rotating cavities with axial throughflow by RANS and LES, in: ASME Turbo Expo 2009: Power for Land, Sea, and Air, American Society of Mechanical Engineers, 2009, pp. 1173-1181.

[12] Q. Tan, J. Ren, H. Jiang, Prediction of 3D unsteady flow and heat transfer in rotating cavity by discontinuous Galerkin method and transition model, in: ASME Turbo Expo 2014: Turbine Technical Conference and Exposition, American Society of Mechanical Engineers, 2014, pp. V05CT16A035V05CT16A035.

[13] J. M. Owen, H. Tang, Theoretical model of buoyancy-induced flow in rotating cavities, Journal of Turbomachinery 137 (11) (2015) 111005.

[17] Z. Sun, A. Kilfoil, J. W. Chew, N. J. Hills, Numerical simulation of natural convection in stationary and rotating cavities, ASME Paper No. GT200453528 . 
[18] D. B. Pitz, J. W. Chew, O. Marxen, N. J. Hills, Direct numerical simulation of rotating cavity flows using a spectral element-fourier method, Journal of Engineering for Gas Turbines and Power 139 (7) (2017) 072602.

[19] D. B. Pitz, O. Marxen, J. W. Chew, Onset of convection induced by centrifugal buoyancy in a rotating cavity, Journal of Fluid Mechanics 826 (2017) 484-502.

[20] D. B. Pitz, J. W. Chew, O. Marxen, N. J. Hills, Large-eddy simulation of buoyancy-induced flow in a sealed rotating cavity, Journal of Engineering for Gas Turbines and Power 141 (2) (2018) 021020.

[21] J. M. Owen, C. A. Long, Review of buoyancy-induced flow in rotating cavities, Journal of Turbomachinery 137 (11) (2015) 111001.

[22] D. Bohn, E. Deuker, R. Emunds, V. Gorzelitz, Experimental and theoretical investigations of heat transfer in closed gas-filled rotating annuli, Journal of Turbomachinery 117 (1995) 175-183.

[23] S. Dong, G. E. Karniadakis, C. Chryssostomidis, A robust and accurate outflow boundary condition for incompressible flow simulations on severely-truncated unbounded domains, Journal of Computational Physics 261 (2014) 83-105.

[24] H. M. Blackburn, S. Sherwin, Formulation of a Galerkin spectral elementFourier method for three-dimensional incompressible flows in cylindrical geometries, Journal of Computational Physics 197 (2) (2004) 759-778.

[25] G. Karniadakis, S. Sherwin, Spectral/hp element methods for computational fluid dynamics, Oxford University Press, 2013.

[26] G. E. Karniadakis, M. Israeli, S. A. Orszag, High-order splitting methods for the incompressible Navier-Stokes equations, Journal of Computational Physics 97 (2) (1991) 414-443. 
[27] G. Karamanos, G. E. Karniadakis, A spectral vanishing viscosity method for large-eddy simulations, Journal of Computational Physics 163 (1) (2000) $22-50$.

[28] R. Pasquetti, Spectral vanishing viscosity method for large-eddy simulation of turbulent flows, Journal of Scientific Computing 27 (1-3) (2006) 365-375.

[29] K. Koal, J. Stiller, H. Blackburn, Adapting the spectral vanishing viscosity method for large-eddy simulations in cylindrical configurations, Journal of Computational Physics 231 (8) (2012) 3389-3405.

[30] J. Lloyd, W. Moran, Natural convection adjacent to horizontal surface of various planforms, Journal of Heat Transfer 96 (4) (1974) 443-447. 


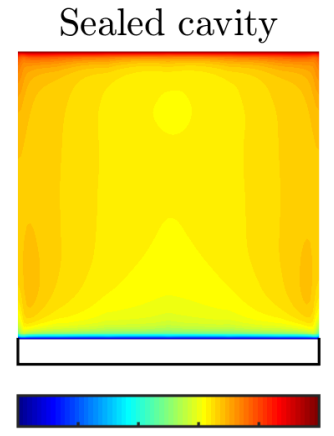

$\begin{array}{llllll}0 & 0.2 & 0.4 & 0.6 & 0.8 & 1\end{array}$

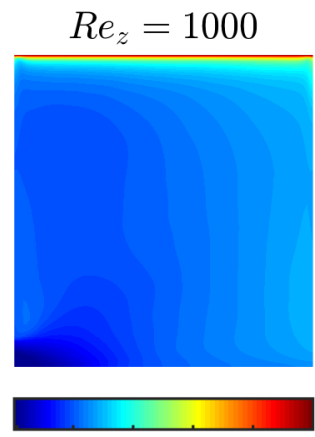

$\begin{array}{lllllll}0 & 0.2 & 0.4 & 0.6 & 0.8 & 1\end{array}$

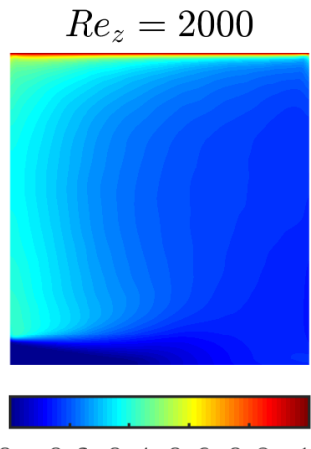

$\begin{array}{lllllll}0 & 0.2 & 0.4 & 0.6 & 0.8 & 1\end{array}$

(a) Temperature $T^{*}$
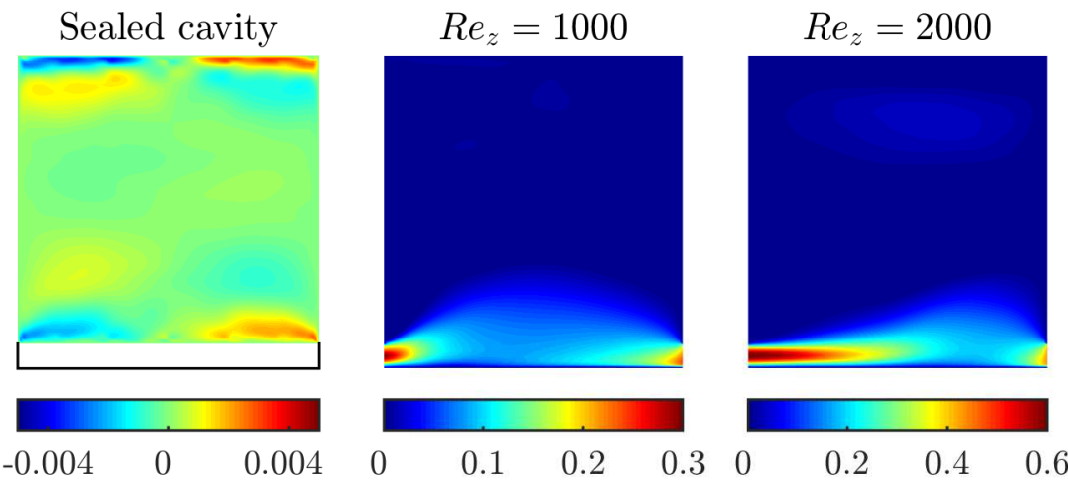

(b) Axial velocity $u_{z}^{\star}$

Figure 6: Time- and circumferentially-averaged contours of (a) normalised temperature and (b) normalised axial velocity, for the case of adiabatic disks. 


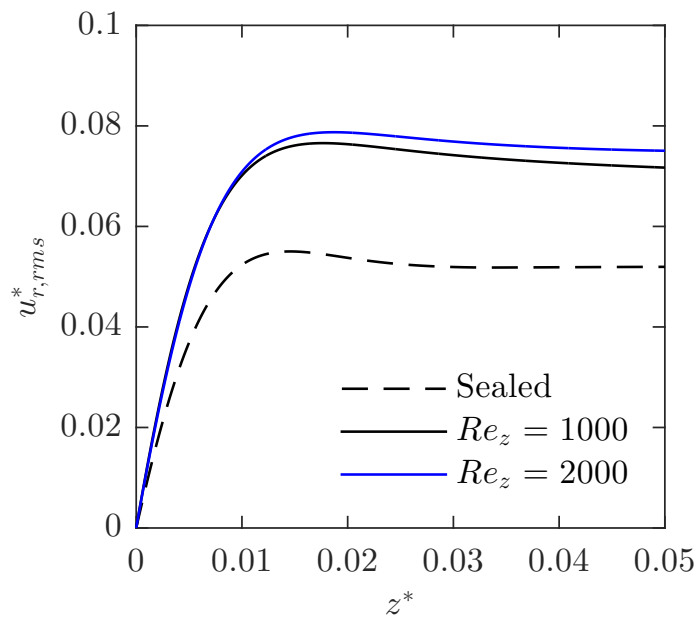

(a) $u_{r, r m s}^{*}$ at $r^{*}=0.5$

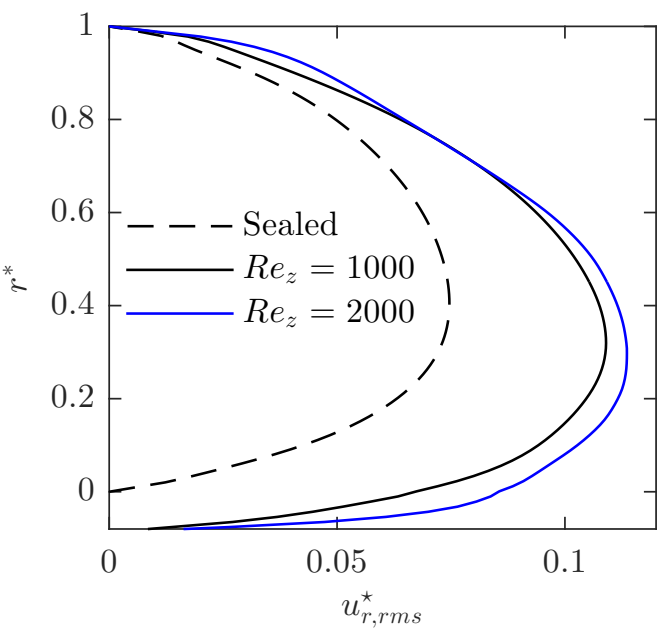

(b) $u_{r, r m s}^{*}$ averaged along $z$

Figure 7: Time- and circumferentially-averaged profiles of the radial velocity fluctuation $u_{r, r m s}^{*}$ (normalised with $\Omega r$ ) and $u_{r, r m s}^{\star}$ (normalised with $\Omega a$ ) for the case of adiabatic disks. The profiles in (a) were extracted at $r^{*}=0.5$, and those of (b) correspond to an average along the axial direction $z$.

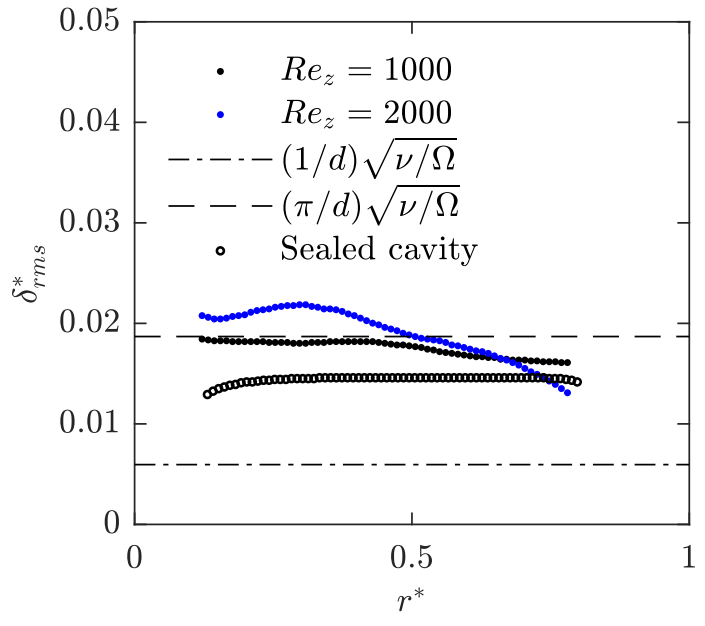

(a) Upstream disk

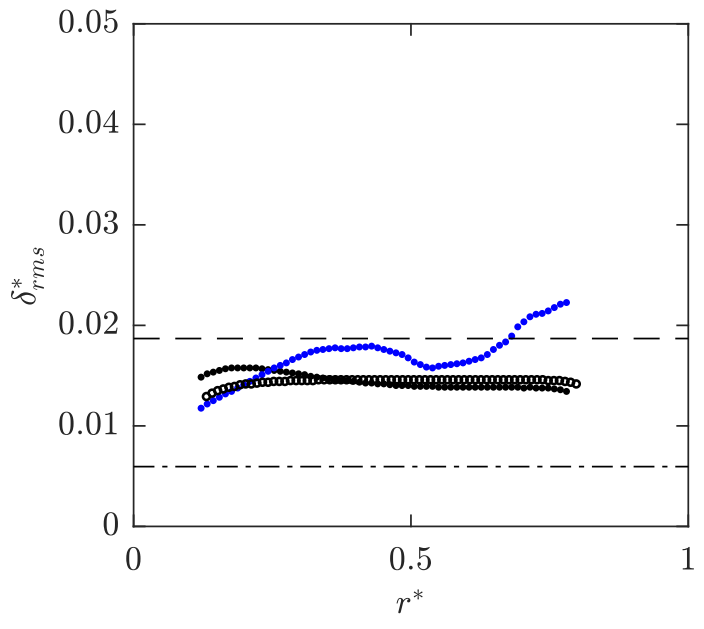

(b) Downstream disk

Figure 8: Radial variation of the disk kinetic boundary layer thickness based on the peak of $u_{r, r m s}^{*}$, for the (a) upstream and (b) downstream disks. 

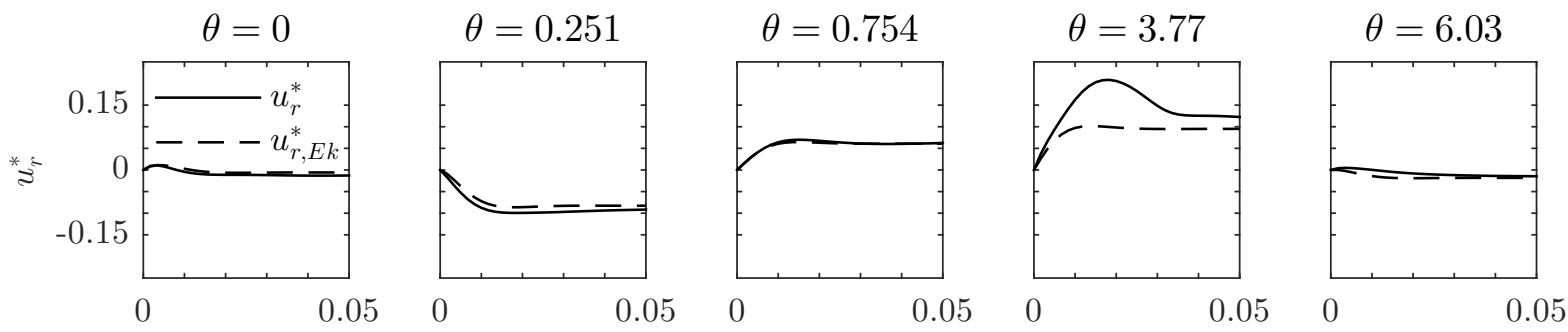

(a) Radial velocity, $\operatorname{Re}_{z}=1000$
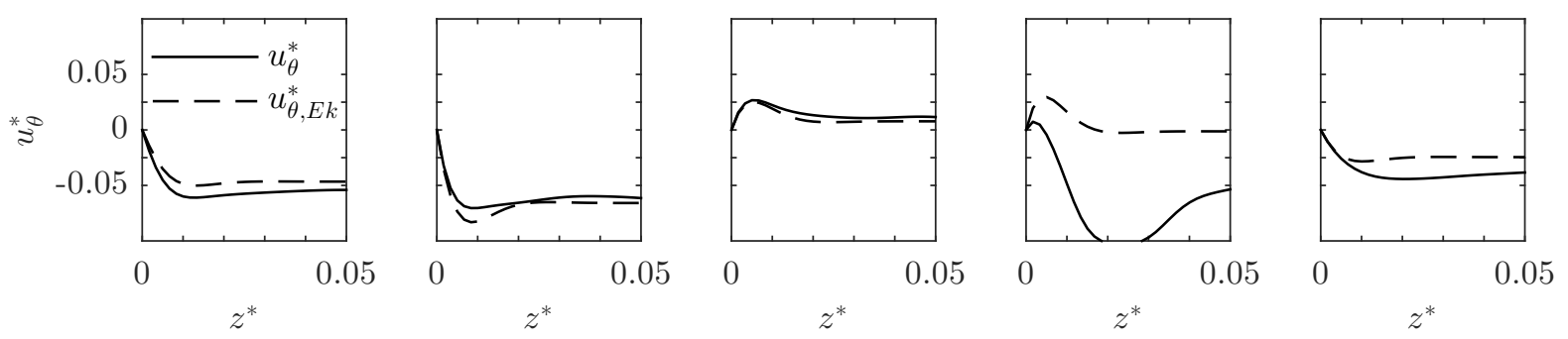

(b) Tangential velocity, $R e_{z}=1000$
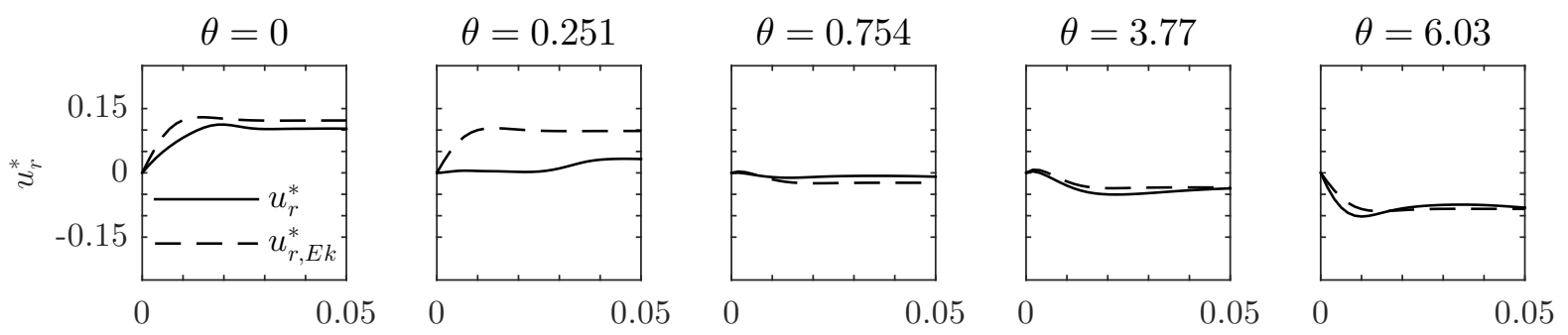

(c) Radial velocity, $R e_{z}=2000$
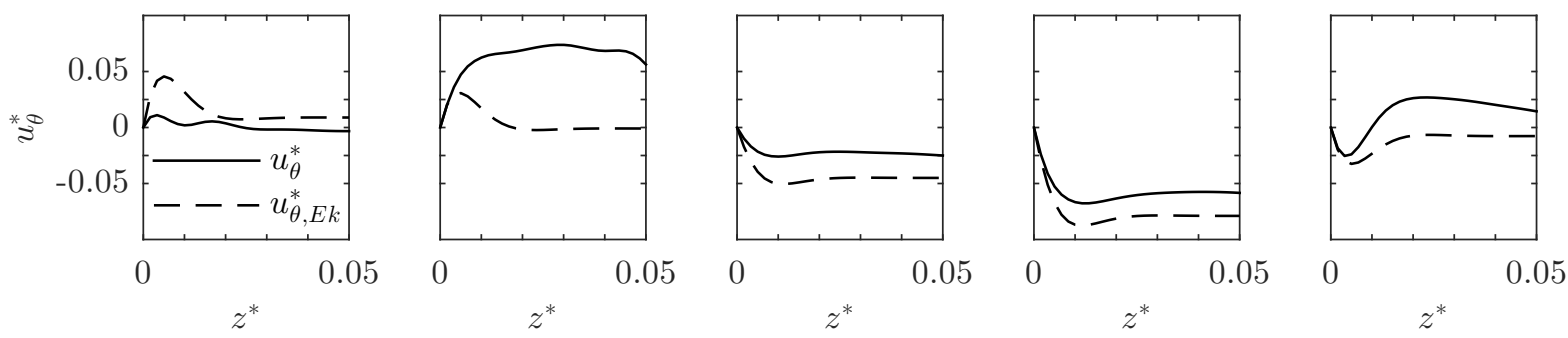

(d) Tangential velocity, $R e_{z}=2000$

Figure 9: Instantaneous profiles of $(\mathrm{a}, \mathrm{c})$ radial and $(\mathrm{b}, \mathrm{d})$ tangential velocity at $r^{*}=0.5$ near the upstream disk, for $(\mathrm{a}, \mathrm{b}) R e_{z}=1000$ and $(\mathrm{c}, \mathrm{d}) R e_{z}=2000$, with adiabatic disks. The dashed lines indicate the laminar Ekman solutions introduced in Eqs. 5 and 6 

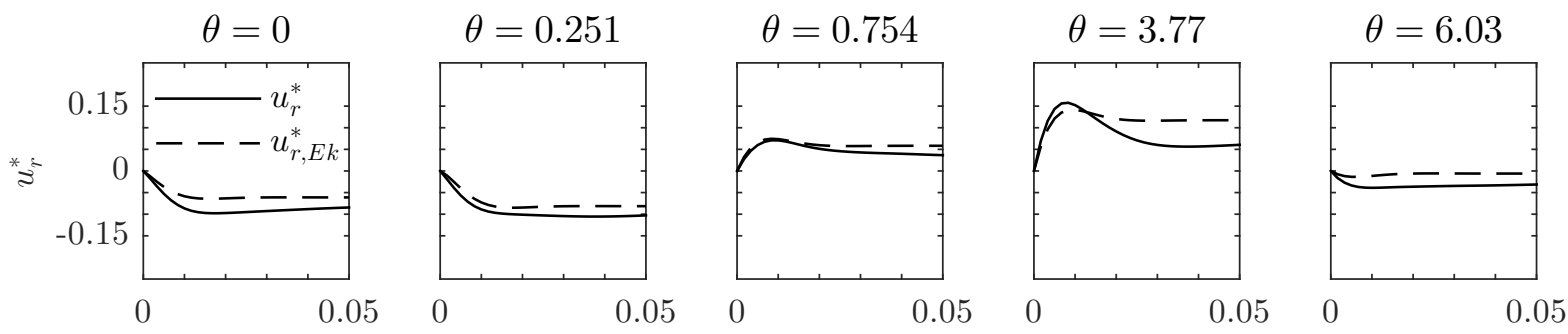

(a) Radial velocity, $R e_{z}=1000$
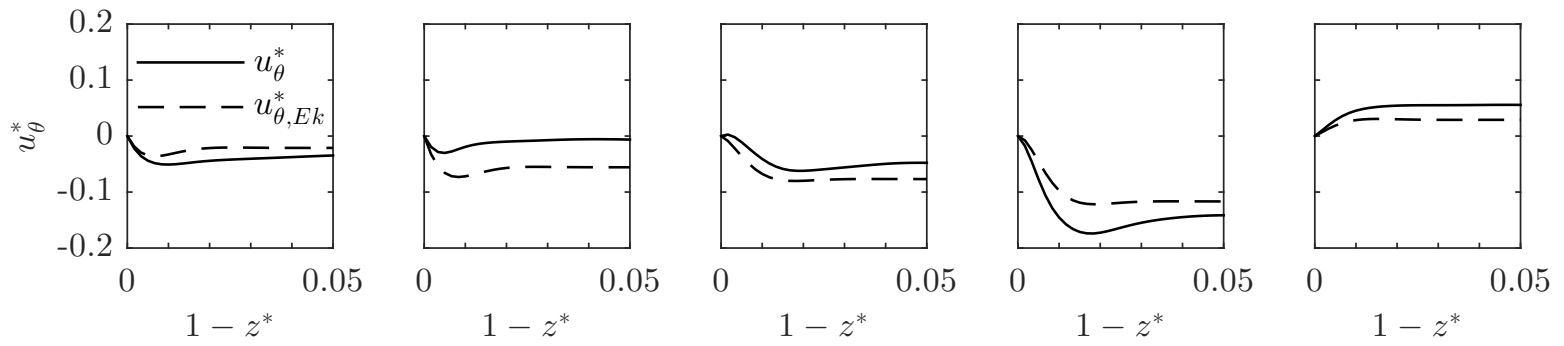

(b) Tangential velocity, $R e_{z}=1000$
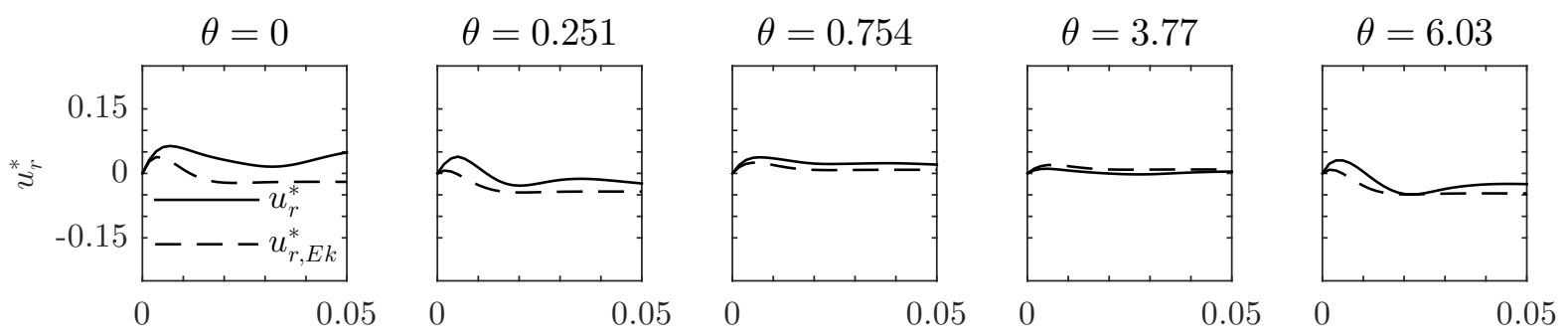

(c) Radial velocity, $R e_{z}=2000$
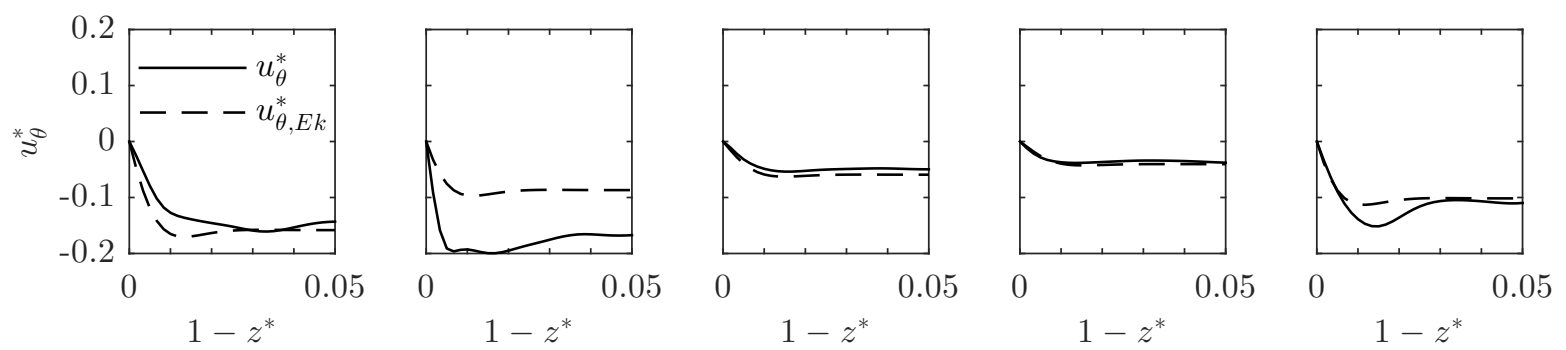

(d) Tangential velocity, $R e_{z}=2000$

Figure 10: Instantaneous profiles of (a,c) radial and (b,d) tangential velocity at $r^{*}=0.5$ near the downstream disk, for (a,b) $R e_{z}=1000$ and (c,d) $R e_{z}=2000$, with adiabatic disks. The dashed lines indicate the laminar Ekman solutions introduced in Eqs. 5 and 6 


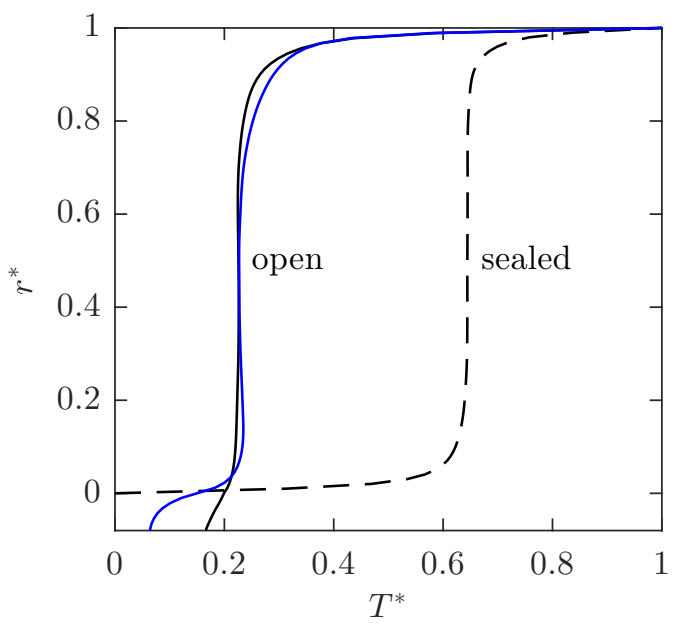

(a) Temperature

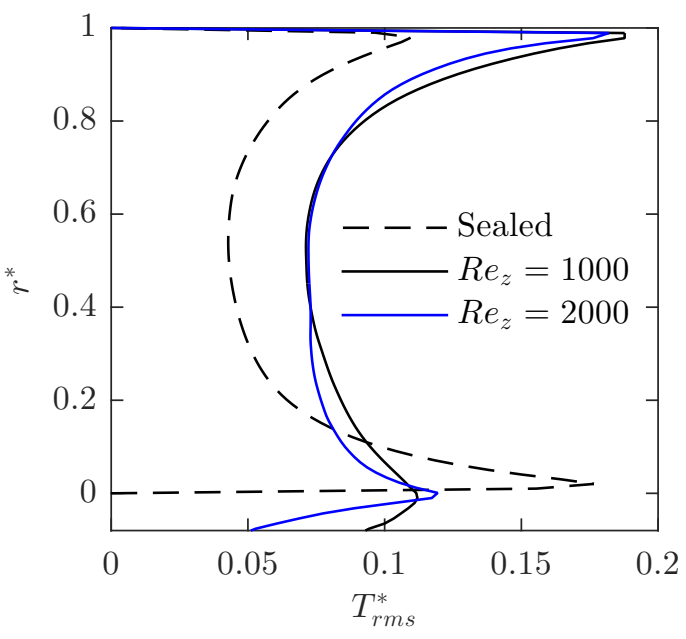

(b) Temperature fluctuation

Figure 11: Time- and circumferentially-averaged profiles of (a) temperature and (b) temperature fluctuation, for the case of adiabatic disks. The profiles were also averaged along the axial extent of the cavity, $0 \leqslant z^{*} \leqslant 1$. 


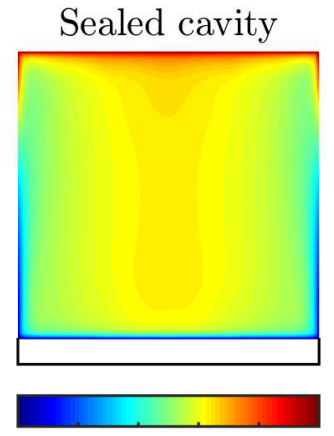

$\begin{array}{llllll}0 & 0.2 & 0.4 & 0.6 & 0.8 & 1\end{array}$

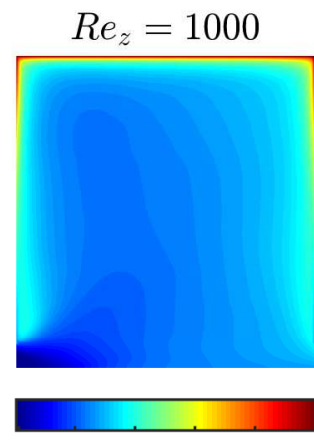

$\begin{array}{llllll}0 & 0.2 & 0.4 & 0.6 & 0.8 & 1\end{array}$

(a) Temperature $T^{*}$
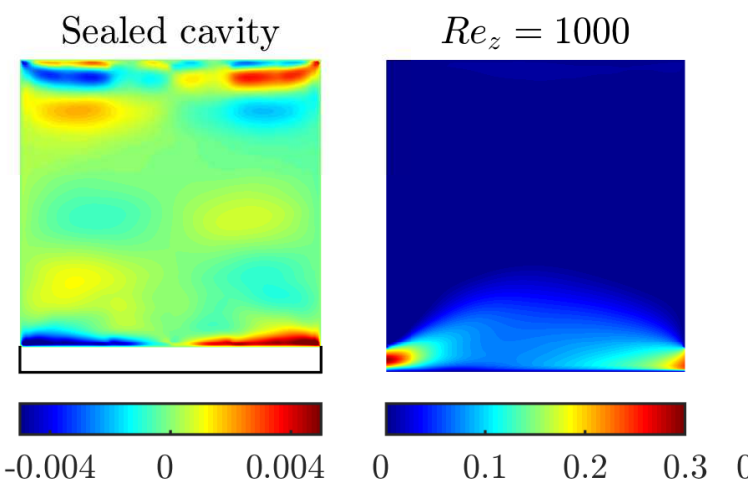

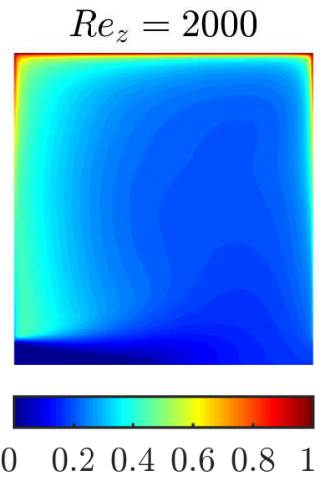

$\begin{array}{llllll}0 & 0.2 & 0.4 & 0.6 & 0.8 & 1\end{array}$

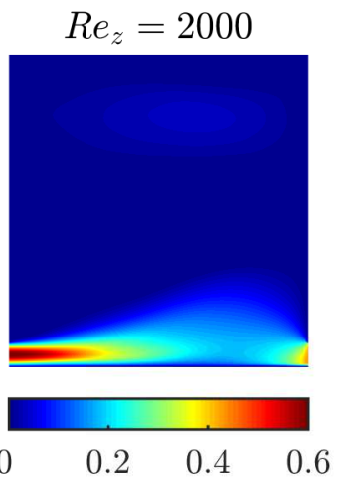

(b) Axial velocity $u_{z}^{\star}$

Figure 12: Time- and circumferentially-averaged contours of (a) normalised temperature and (b) normalised axial velocity, for the case of heated disks. 


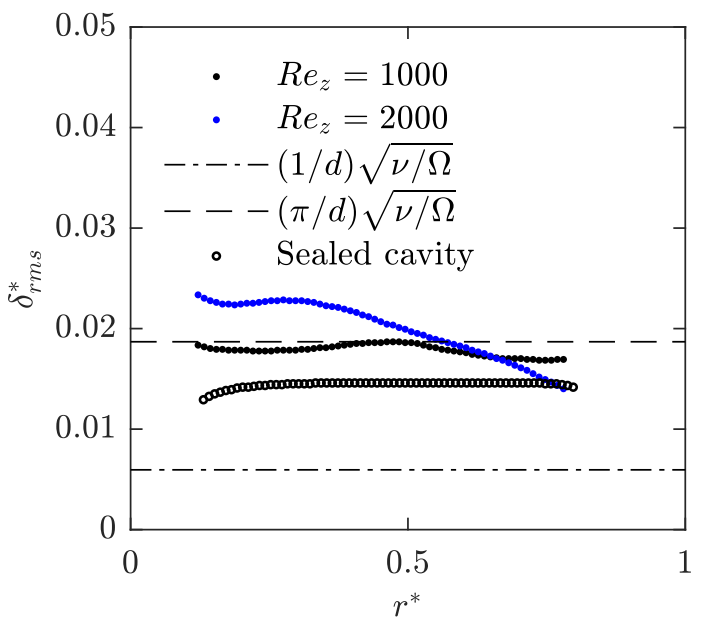

(a) Upstream disk

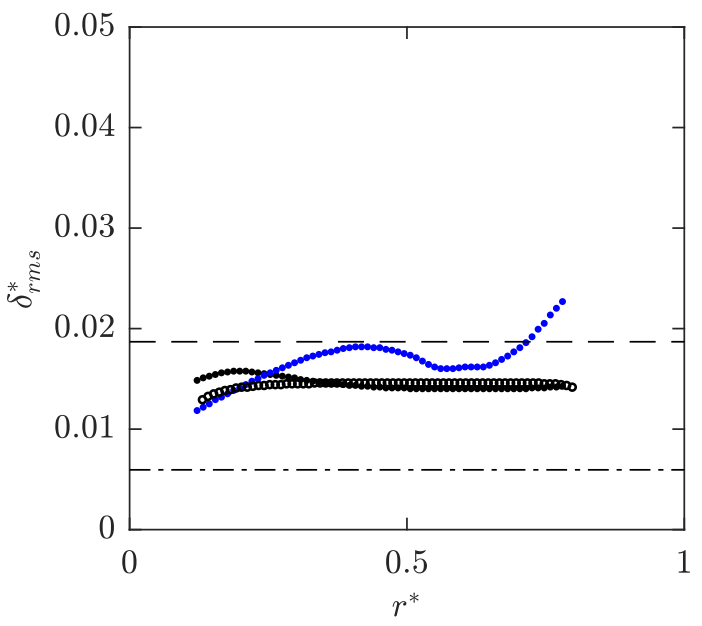

(b) Downstream disk

Figure 13: Radial variation of the disk kinetic boundary layer thickness based on the peak of $u_{r, r m s}^{*}$ for the case of heated disks, for the (a) upstream and (b) downstream disks.

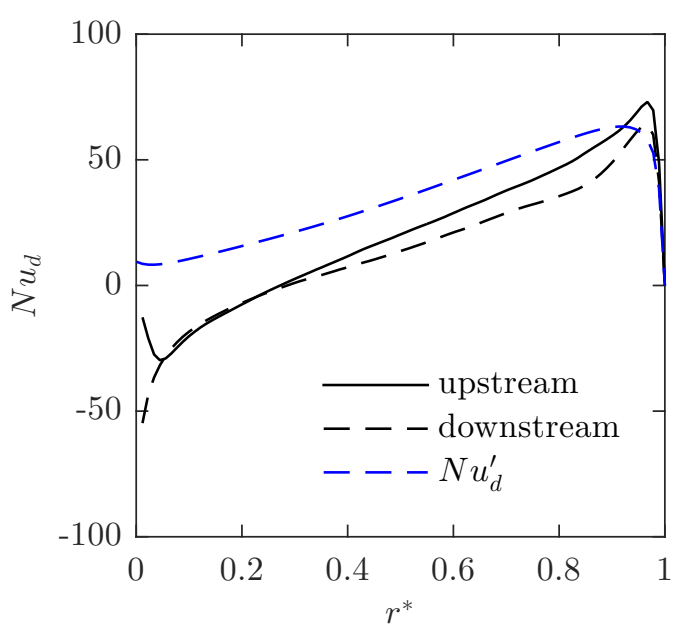

(a) $R e_{z}=1000$

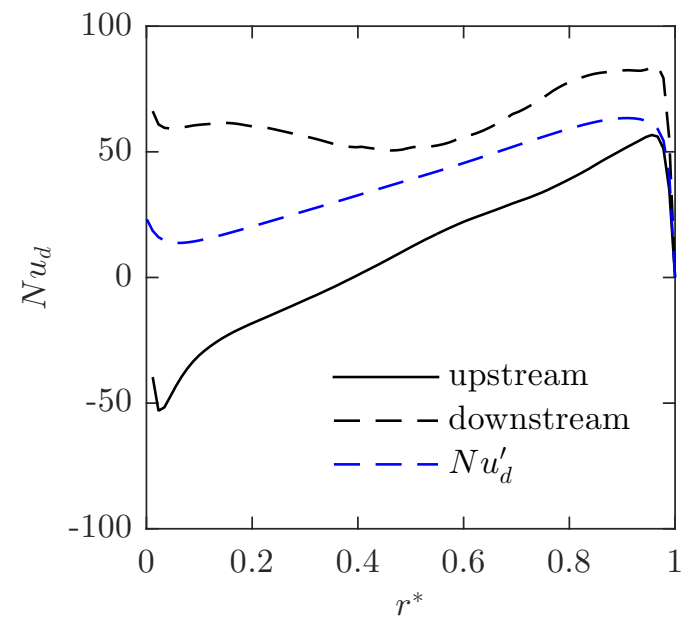

(b) $R e_{z}=2000$

Figure 14: Profiles of the disk Nusselt number $N u_{d}$ as a function of the radial position, for both the upstream and downstream disks. 

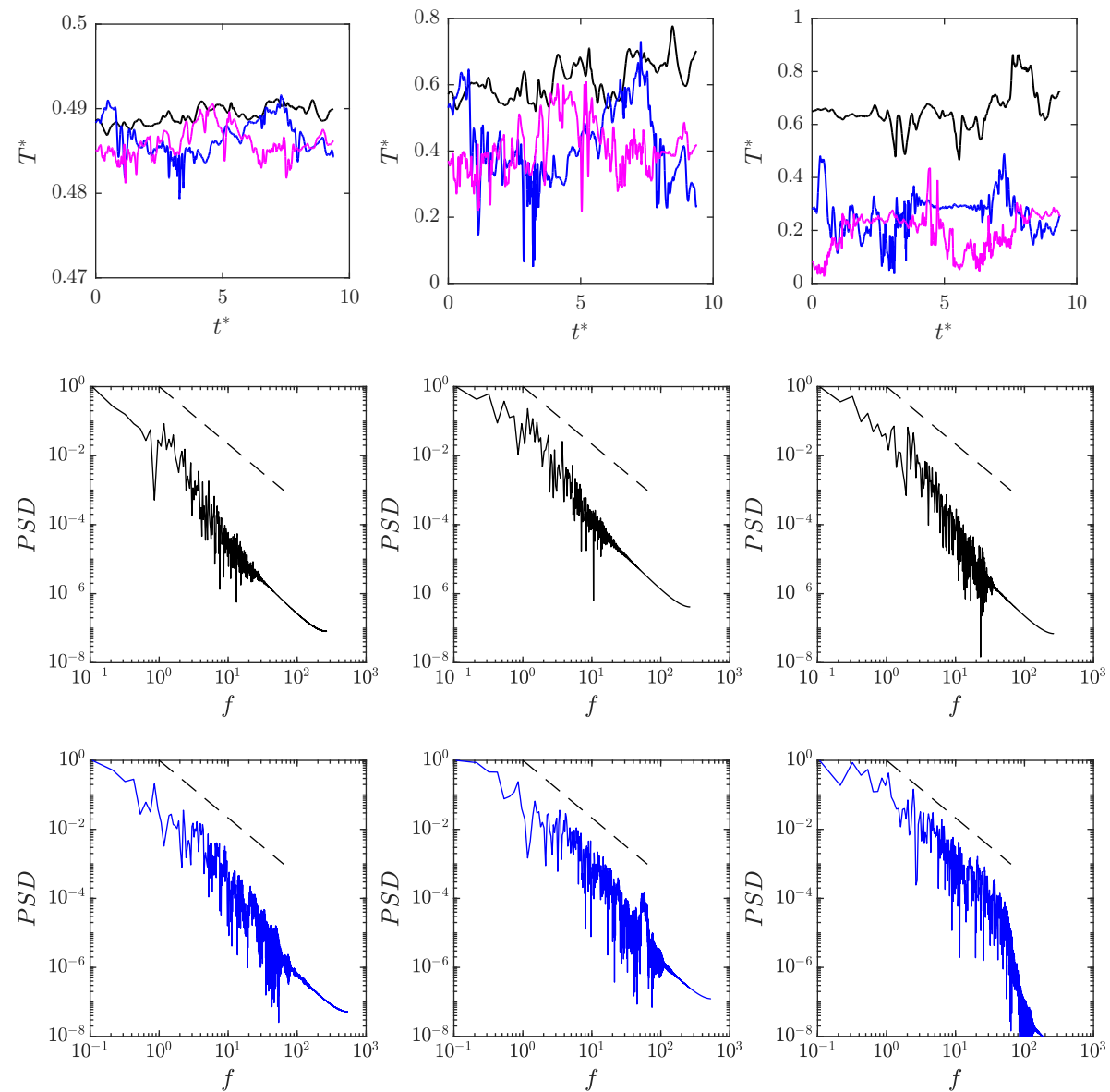

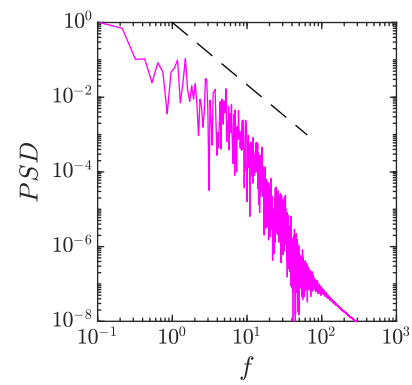

(a)

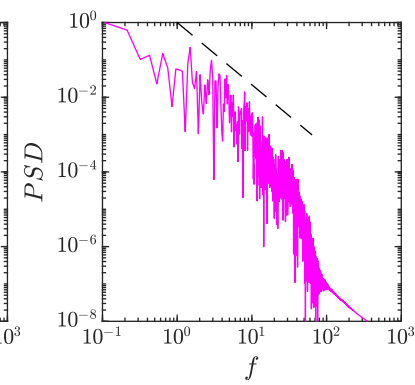

(b)

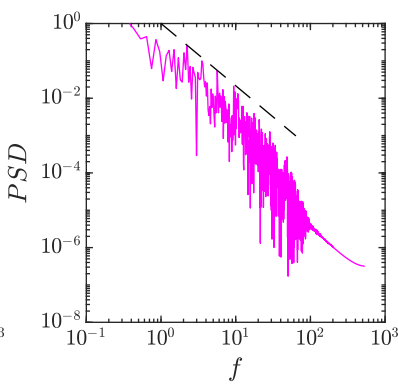

(c)

Figure 15: Normalised temperature $T^{*}$ as a function of $t^{*}$ (top row) for the sealed cavity (black), $R e_{z}=1000$ (blue) and $R e_{z}=2000$ (magenta) and corresponding power spectral densities, where the frequency is normalised by $\Omega / 2 \pi$, for (a) $z^{*}=2 \times 10^{-4}$, (b) $z^{*}=0.02$, (c) $z^{*}=0.5$. In all cases $r^{*}=0.5$ and $\theta=0$. The dashed lines indicate a $f^{-5 / 3}$ slope. 

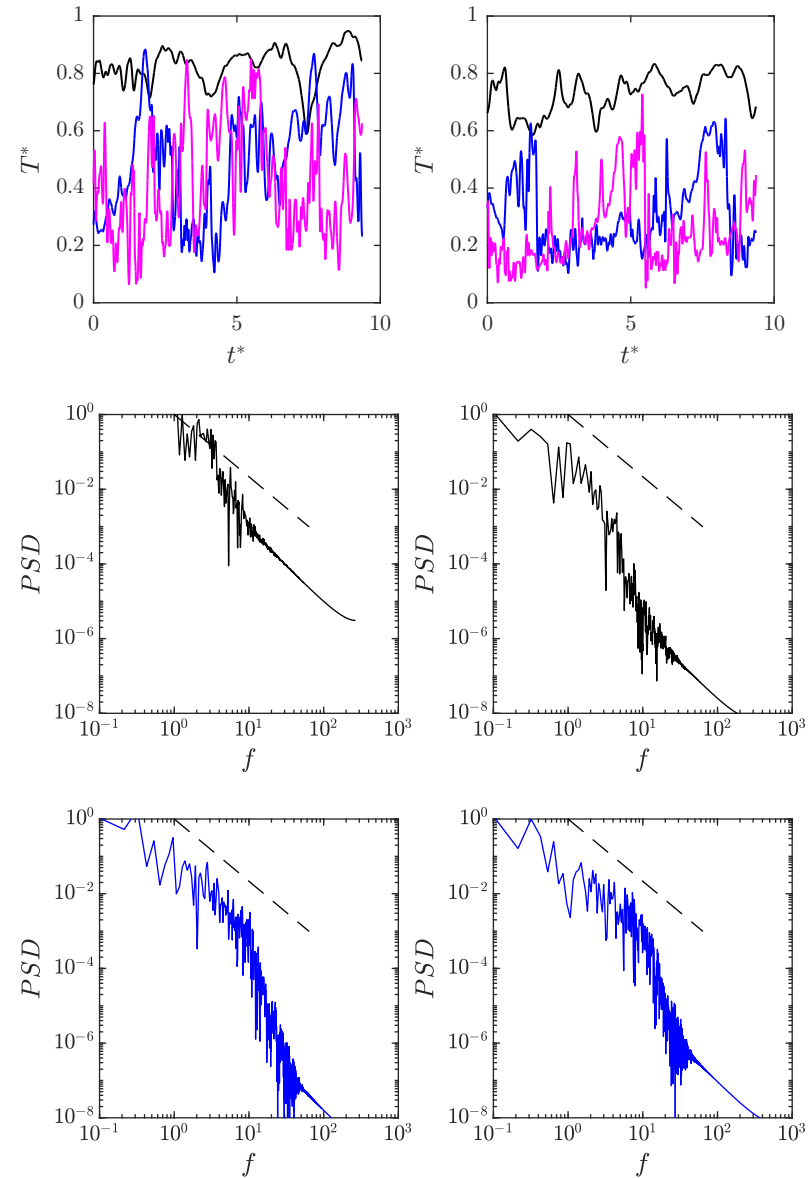

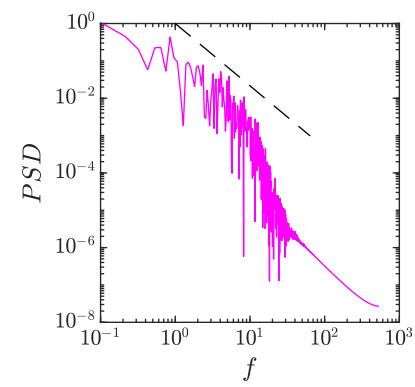

(a)

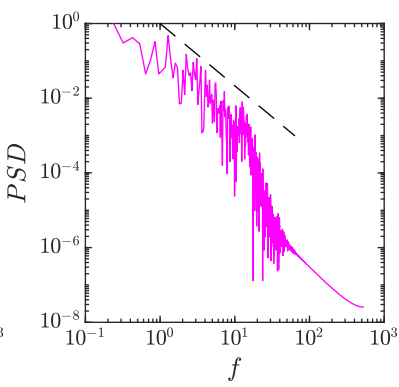

(b)

Figure 16: Normalised temperature $T^{*}$ as a function of $t^{*}$ (top row) for the sealed cavity (black), $R e_{z}=1000$ (blue) and $R e_{z}=2000$ (magenta) and corresponding power spectral densities, where the frequency is normalised by $\Omega / 2 \pi$, for (a) $r^{*}=0.98$ and (b) $r^{*}=0.9$. In all cases $z^{*}=0.5$ and $\theta=0$. The dashed lines indicate a $f^{-5 / 3}$ slope. 


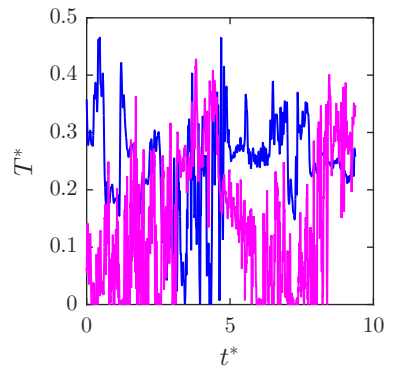

(a)

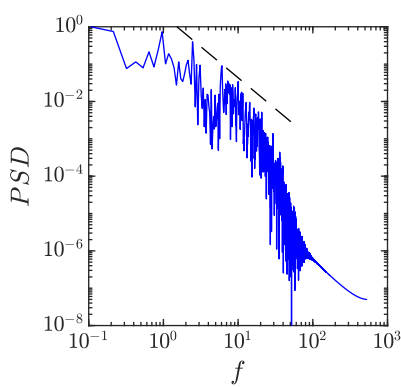

(b)

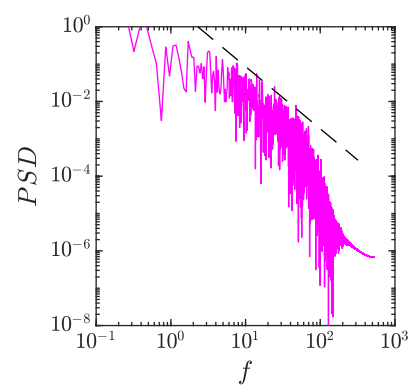

(c)

Figure 17: (a) Normalised temperature $T^{*}$ as a function of $t^{*}$ for $R e_{z}=1000$ (blue) and $R e_{z}=2000$ (magenta) at $r^{*}=0.04, z^{*}=0.5$ and $\theta=0.0$. (b,c) Corresponding power spectral densities, where the frequency is normalised by $\Omega / 2 \pi$. The dashed lines indicate a $f^{-5 / 3}$ slope. 